\title{
Changes in plasma cortisol and catecholamine concentrations in response to massage in preterm infants
}

\author{
D Acolet, N Modi, X Giannakoulopoulos, C Bond, W Weg, A Clow, V Glover
}

\begin{abstract}
The biochemical and clinical response to massage in preterm infants was assessed. Eleven stable infants, of 29 weeks' median gestational age, median birth weight 980 $\mathrm{g}$, and median postnatal age 20 days, were studied. Blood samples were obtained for the determination of adrenaline, noradrenaline, and cortisol 45 minutes before the start of massage and approximately one hour after completion of massage. Cortisol, but not catecholamine, concentrations decreased consistently after massage (median difference $-35.8 \mathrm{nmol} / \mathrm{h} ; 95 \%$ confidence interval -0.5 to $-94 \cdot 0$, Wilcoxon matched pairs). There was a slight decrease in skin temperature (median difference $-0.36^{\circ} \mathrm{C}, 95 \%$ confidence interval -0.09 to -0.65 ) but there was no change in oxygenation or oxygen requirement. This study has shown that it is possible to detect an objective hormonal change following a supposedly 'non-therapeutic' intervention in preterm infants. The development of such methods of assessment are likely to be of particular relevance in the extremely immature or ill neonate in whom behavioural evaluation cannot play more than a limited part.
\end{abstract}

(Arch Dis Child 1993;68:29-31)

Department of Paediatrics and Neonatal Medicine, Royal Postgraduate Medical School, London

D Acolet

N Modi

C Bond

Department of Chemical Pathology, Queen Charlotte's and Chelsea Hospital, London

$\mathrm{X}$ Giannakoulopoulos

W Weg

A Clow

V Glover

Correspondence to: Dr N Modi,

Department of Paediatrics

and Neonatal Medicine

and Neonatal Medicine, Royal Postgraduate Medical
School, Queen Charlotte's School, Queen Charlote's and Chelsea Hospita

London W6 0XG.

Accepted 29 June 1992 Reprints not available.
Infants admitted to neonatal intensive care units are exposed to a number of stresses. Neonatal units are often noisy and overbright; the infants often require painful procedures and may have painful disorders; they may undergo metabolic stress as a consequence of their underlying illness; and we do not know whether they experience the stress of anxiety or fear. There is evidence that even preterm infants can mount a strong biochemical response to the stress of an operation with increased concentrations of catecholamines and cortisol. ${ }^{1}$

Positive interventions, aimed at improving the quality of life for preterm infants in intensive care units, include the use of sheepskins, mattresses filled with polystyrene balls, massage, and music. These interventions have rarely been evaluated objectively, though one of us (DA) has previously shown motherinfant skin to skin contact to be beneficial. ${ }^{2}$ chemical and clinical response to massage in preterm infants.
The aim of this study was to assess the bio-
Patients and methods

Eleven preterm infants nursed in the neonatal intensive care unit at Queen Charlotte's and Chelsea Hospital were evaluated before and after massage. The infants were clinically stable and the massage was carried out in the late afternoon by an experienced neonatal nurse (CB) who has used the technique for several years. It consists of gentle massage of the trunk and limbs using arachis oil (fig 1) and lasts approximately 20 minutes.

A blood sample $(0 \cdot 6-0 \cdot 8 \mathrm{ml})$ was obtained 45 minutes before the start of massage and approximately one hour after completion of massage to determine adrenaline, noradrenaline, and cortisol. The blood sample was collected into heparinised tubes, spun immediately at $600 \mathrm{~g}$ for five minutes, and the plasma was separated and stored at $-70^{\circ} \mathrm{C}$ until assayed. Skin temperature, oxygen saturation, transcutaneous oxygen tension, and, for infants requiring additional oxygen, the change in oxygen requirement to maintain oxygen saturation between 92 and $96 \%$ were recorded throughout the approximately four hour study period. To determine whether any variation in cortisol concentrations was due to diurnal variation, paired blood samples were also obtained, in the late afternoon, from eight stable control infants matched for gestational and postnatal age.

Cortisol was determined in plasma by radioimmunoassay ${ }^{3}$ and adrenaline and noradrenaline by radioenzymatic assay. ${ }^{4}$ Nonparametric tests were used for data analysis. The study was approved by the ethics committee of the Royal Postgraduate Medical School and Hammersmith and Queen Charlotte's Special Health Authority. Consent was obtained from the parents.

\section{Results}

Eleven boys were studied. They remained stable throughout the study period. Gestational age ranged from 23 to 34 weeks (median 29 weeks), birth weight from 630 to $2180 \mathrm{~g}$ (median $980 \mathrm{~g}$ ), and postnatal age from 4 to 132 days (median 20 days). Six infants were receiving supplemental oxygen.

In three samples insufficient plasma was obtained to determine cortisol. Figure 2 shows the change in plasma cortisol concentration in the eight infants in whom paired samples were obtained. The table gives the range and median 


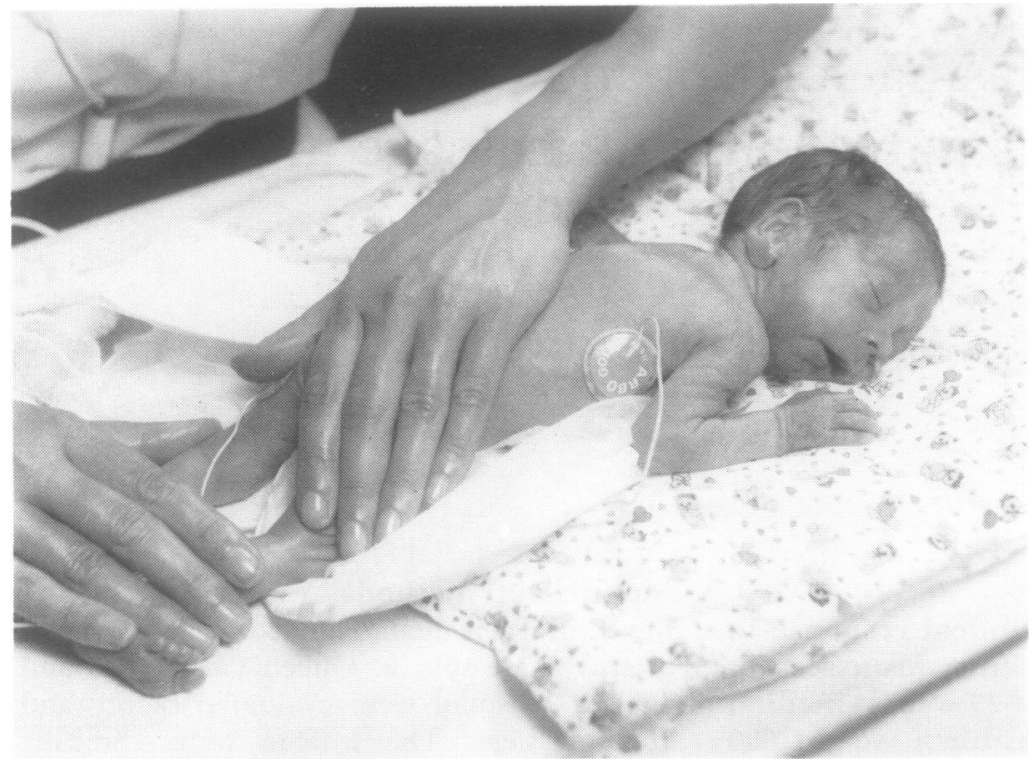

Figure 1 Preterm infant being massaged.

values for cortisol, adrenaline, and noradrenaline before and after massage. Cortisol concentrations were consistently reduced after massage. Although the calculated median difference was $-35.8 \mathrm{nmol} / \mathrm{l}$ ( $95 \%$ confidence interval -0.5 to $-94.0 ; \mathrm{p}=0.035$, Wilcoxon matched pairs), the actual difference was even greater than this, as four of the eight readings after massage were below the limit of detection of $30 \mathrm{nmol} / 1$. Adrenaline (fig 3) and noradrenline (fig 4) concentrations showed no consistent change $(95 \%$ confidence interval for change in adrenaline -0.168 to $0.011 ; \mathrm{p}=0.24$, $95 \%$ confidence interval for change in noradrenaline $-0 \cdot 18$ to $0 \cdot 13 ; p=0.92$ ). There was no significant change in oxygenation or oxygen

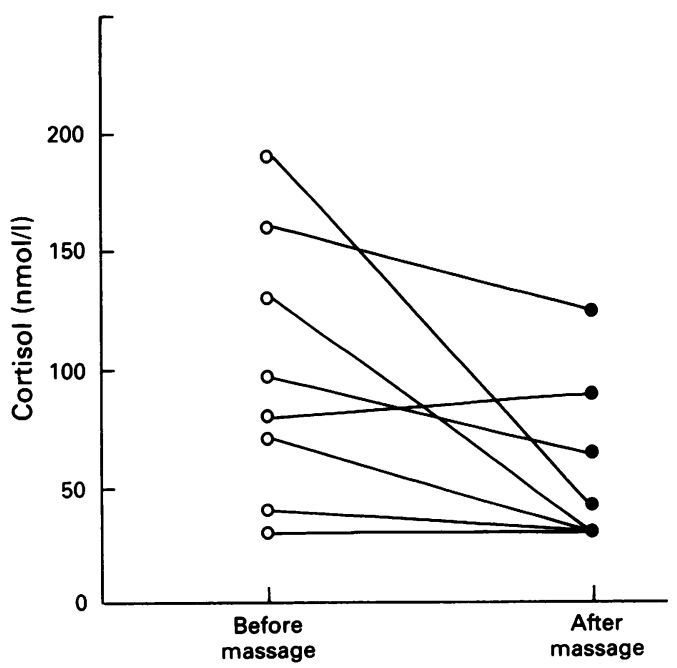

Figure 2 Effect of massage on plasma cortisol concentrations.

Range (median) values for cortisol and catecholamines concentrations in preterm infants before and after massage

\begin{tabular}{|c|c|c|}
\hline & $\begin{array}{l}\text { Before } \\
\text { massage }\end{array}$ & $\begin{array}{l}\text { After } \\
\text { massage }\end{array}$ \\
\hline $\begin{array}{l}\text { Cortisol }(\mathrm{nmol} / \mathrm{l}) \\
\text { Adrenaline }(\mathrm{ng} / \mathrm{ml}) \\
\text { Noradrenaline }(\mathrm{ng} / \mathrm{ml})\end{array}$ & $\begin{array}{l}<30-190(89)(n=10) \\
<0.01-0.47(0.039)(n=10) \\
0.35-1.380(0.69)(n=10)\end{array}$ & $\begin{array}{l}<30-125(<30)(n=9) \\
<0.01-0.11(0.021)(n=10) \\
0.32-1 \cdot 16(0.66)(n=10)\end{array}$ \\
\hline
\end{tabular}

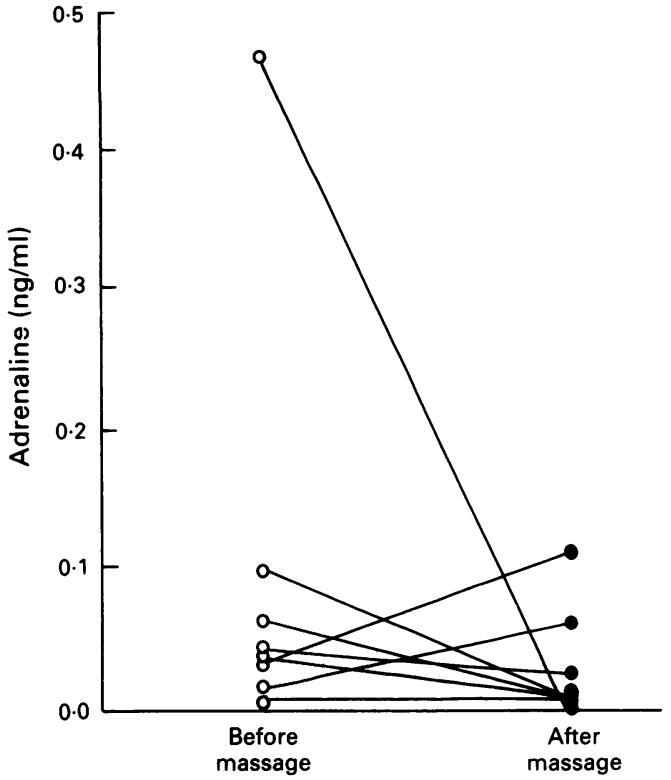

Figure 3 Plasma adrenaline concentrations in massaged infants $(n=10)$

requirement before, during, and after massage. There was a slight decrease in skin temperature (median difference $-0.36^{\circ} \mathrm{C}, 95 \%$ confidence interval -0.09 to -0.65 ).

There was no significant difference in weight, gestational age, and postnatal age between the infants receiving massage and control infants (Mann-Whitney $U$ test). Paired samples for cortisol determination obtained from controls, at the same time of day and over the same time interval as the infants receiving massage, showed no consistent change $(95 \%$ confidence interval -85 to $223 ; p=0 \cdot 44)$. No significant difference was detected between the change in cortisol concentration in the group receiving massage and in the control group ( $95 \%$ confidence interval -226 to $45 ; \mathrm{p}=0 \cdot 13$ ).

\section{Discussion}

Although there has been a surge of interest in

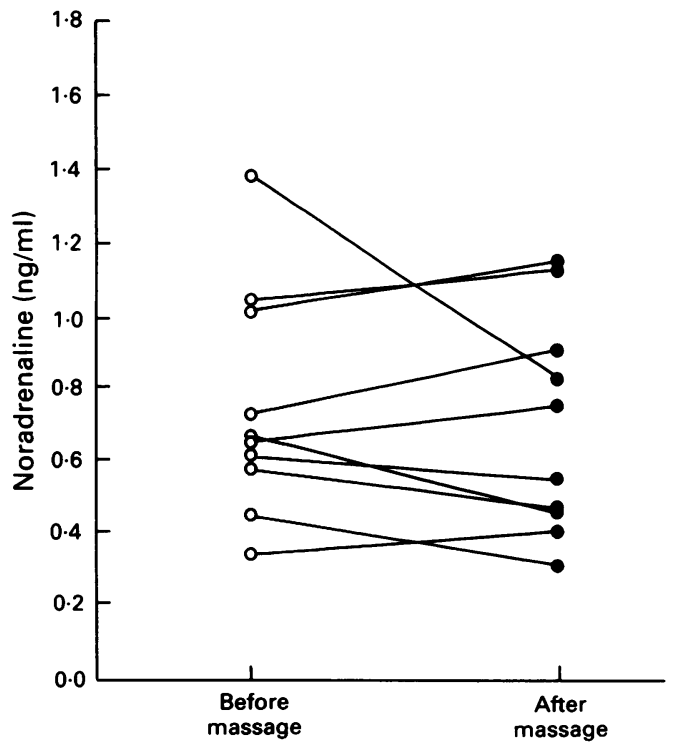

Figure 4 Plasma noradrenaline concentrations in massaged infants $(n=10)$ 
methods of pain relief in neonates, ${ }^{1}$ acute, positive interventions, designed to improve the quality of life for preterm infants receiving intensive care have rarely been objectively evaluated. Behavioural assessment is often subjective and open to observer bias; in the most ill infants behavioural assessment is not possible and physiological assessment is difficult because of the wide variation in clinical conditions. Tactile sensibility is the first of the human senses to appear and tactile stimulation programmes of various kinds have been reported to influence the long term outcome with improved neurodevelopmental maturation and growth. ${ }^{5}$ To our knowledge, however, no immediate benefits have been described.

We have now provided evidence of an immediate response to massage, showing a consistent decrease in plasma cortisol concentrations. This is unlikely to be due to diurnal variation as no such difference was seen in the control infants.

The stress of pain results in an increase in cortisol concentrations ${ }^{1}$; the response to massage was the converse. It is of interest that we found no consistent change in the concentration of either catecholamine. Our findings mirror those of a study in adults which showed a decrease in serum cortisol but no significant change in catecholamines following a reduction in stress induced by laughter. ${ }^{6}$ The relation between the different chemical stress responses of the body is only partially understood, but much is currently being done to try to disentangle them. In adults plasma cortisol concentrations have been found to correlate with so called vegetative symptoms, such as insomnia and weight loss, rather than with cognitive symptoms such as guilt. ${ }^{7} \mathrm{We}$ confirmed a small decrease in skin tempera- ture during massage; this is already recognised in everyday clinical practice as ambient room temperature is normally routinely increased during massage.

We feel that it would be premature for us to imply, on the basis of our data, that massage is either beneficial or pleasurable. Furthermore, the wide confidence interval for the decrease in cortisol concentration suggests that we should regard these results as the justification for further evaluation. We have, however, shown that it is possible to detect an objective hormonal change following a supposedly 'non-therapeutic' intervention in preterm infants. The development of such methods of assessment is likely to be of particular relevance in the extremely immature or ill neonate, in whom behavioural evaluation cannot play more than a limited part. With such tools we may, in addition to improving long term outcome, be better able to make neonatal intensive care a more humane practice.

1 Anand KJS, Hickey PR. Pain and its effects on the human fetus and neonate. $N$ Engl F Med 1987;317:1321-9.

2 Acolet $D$, Sleath $K$, Whitelaw $A$. Oxygenation, heart rate and temperature in very low birth weight infants during skin to skin contact with their mothers. Acta Paediatr Scand 1989;78:189-93.

3 Donaldson A, Nicolini U, Symes EK, Rodeck $\mathrm{CH}$, Tannirandorn Y. Changes in concentrations of cortisol, dehydroepiandrosterone sulphate and progesterone in fetal and maternal serum during pregnancy. Clin Endocrinol (Oxf) 1991;35:1-5.

4 Brown MJ, Jenner DA. Novel double-isotope technique for enzymatic assay of catecholamines, permitting high precision, sensitivity and plasma sample capacity. Clin Sci $1981 ; 61: 591-8$.

5 Adamson-Macedo EN. The effects of touch on preterm and fullterm neonates and young chidlren. Fournal of Reproduction and Infant Psychology 1990;8:267-73.

6 Berk LS, Tan SA. Fry WF, et al. Neuroendocrine and stress hormone changes during mirthful laughter. Am $\mathcal{f}$ Med Sci 1989;298:390-6.

7 Faustman WD, Faull KF, Whiteford HA, Borchert C, Csernansky JG. Cerebrospinal fluid 5HIAA, serum cortisol and age differentially predict vegetative and cognitive symptoms in depression. Biol Psychiatry 1990;27:311-8. 